\title{
Collection and Characterization of Indigenous Dioscorea bulbifera Genotypes of Chhattisgarh
}

\author{
Kamal Narayan ${ }^{1 *}$, J. Singh ${ }^{2}$, Deo Shankar ${ }^{2}$, R. Gayen ${ }^{3}$, N. Mehta ${ }^{4}$ and R. R. Saxena ${ }^{5}$ \\ ${ }^{1}$ Horticulture, ${ }^{2}$ Department of floriculture and landscape Architecture), ${ }^{3}$ Department of \\ Vegetable science, ${ }^{4}$ Department of Genetics and Plant Breeding), KVK, Pahanda (A), Durg, \\ I.G.K.V. (C.G.), India \\ ${ }^{5}$ Director of Research, IGKV, Raipur, India \\ *Corresponding author
}

\section{A B S T R A C T}

\begin{tabular}{|l|}
\hline Key w ord s \\
$\begin{array}{l}\text { Dioscorea } \\
\text { bulbifera, } \\
\text { Cordate leaf shape }\end{array}$ \\
\hline Article Info \\
\hline $\begin{array}{l}\text { Accepted: } \\
26 \text { August } 2020 \\
\text { Available Online: } \\
\text { 10 September } 2020\end{array}$ \\
\hline
\end{tabular}

Thirty indigenous genotypes of Dioscorea (Dioscorea bulbifera) collected from twelve districts of Chhattisgarh viz., Bastar, Korba, Dhamtari, Rajnandgaon, Surguja, Kondagaon, Sukma, Balod, Narayanpur, Kanker, Dantewada and Bijapur. The collected genotypes show wide range of variability in morphological characteristics. More variability was recorded in genotypes collected from Sukma district of Chhattisgarh followed by Bijapur and Kanker district. Petiole colour varied from all green with purple at both ends and all purplish green with purple at both ends with the frequency of $26.67 \%$ and $73.33 \%$ respectively. Petiole length in correlation to leaf blade is characterized in to three categories i.e. short, medium and long with $13.33 \%, 13.33 \%$ and $73.33 \%$ respectively. Highest frequency $(50 \%)$ was recorded as rough aerial tuber texture followed by wrinkled (40\%) and lowest frequency (10\%) as smooth. However, all the genotypes of Dioscorea bulbifera exhibits cordate leaf shape, opposite leaf position, entire leaf margin, acute apex shape and the distance between lobes is intermediate.

\section{Introduction}

Dioscorea is a large genus of annual twining herbs, distributed throughout the moist tropics of the world and extending into warm temperate regions. About 50 species are found in India; a large number among them occur in the wild state and a few are cultivated for their edible tubers. The yams of several species are soft, fleshy and edible. In Chhattisgarh, aerial yam has been cultivated mainly and extensively in densely populated and high rainfall areas. In South part of the state, a large pool of aerial yam germplasm is found in farmer's field and forest areas. However, the existence of different vernacular names for the same cultivar of the species, or vice versa has created problems to classify accessions while avoiding duplicates. Yams form a cheap source of carbohydrate food and are extensively used by hill tribes in the uncultivated tracts of Assam, Bihar, Bengal, Madhya Pradesh, Orissa, Deccan etc. (Annonymous, 2005). Arial yam commonly 
known as Dioscorea bulbifera in India; air potato in abroad and locally known as Dang Kanda, Lathi Kanda and Laat Kanda in Chhattisgarh. It is a rich source of carbohydrate, protein, carotene and other vitamins and has high calorific value. It is a Kharif season crop and is most suitable under rainfed condition. Aerial yam comes under the group of minor tuber crop but it has major importance due to ethnic and high market value as compare to other Dioscorea species and it is a stable food of tribal farmers of Chhattisgarh.

Proper characterization and evaluation of germplasm and dissemination of the information to the plant breeders and others is a priority area in any germplasm management programme. Characterization of germplasm consists traits that are highly heritable, expressed in different environments and can be easily seen by the eye. Characterization information along with passport data provides an indication of the range of diversity in the collections, and is of considerable help to the breeders to narrow the selection of potential breeding stocks. Until a collection has been properly evaluated and its attributes become known to breeders, it has little practical use (Thomas and Mathur, 1991).

\section{Materials and Methods}

The present investigation entitled "Collection, and characterization of indigenous Dioscorea bulbifera genotypes of Chhattisgarh" was conducted at IGKV, Shaheed Gundadhoor College of Agriculture \& Research Station (SG CARS), Kumhrawand, Jagdalpur, Bastar, Chhattisgarh during Kharif seasons of 201718 and 2018-19.

The soil situation of the experimental site is silty loam texture with $\mathrm{pH}$ of 6.5 . Nitrogen and potassium content of the soil is low, while phosphorus level is medium to low. Bastar- plateau comes under moist, sub-humid agroclimatic region of Chhattisgarh.

The Bastar division of Chhattisgarh received high rainfall coupled with comparatively lower temperatures and higher humidity. The average annual rainfall of this region is 1380 $\mathrm{mm}$, most of which $(85 \%)$ is received from third week of June to mid September and remaining distributed during February, March, May and October.

Thirty indigenous genotypes of Dioscorea (Dioscorea bulbifera) collected from twelve districts of Chhattisgarh viz., Bastar, Korba, Dhamtari, Rajnandgaon, Surguja, Kondagaon, Sukma, Balod, Narayanpur, Kanker, Dantewada and Bijapur (Table 1).

The experiment consists of 30 genotypes of Dioscorea planted in three replications. The genotypes were grown randomly in each replication/block in a total of 90 plots of $3 \mathrm{~m} \mathrm{x}$ $3 \mathrm{~m}$ each containing 16 plants per plot with a spacing of $75 \mathrm{~cm}$ row to row and $75 \mathrm{~cm}$ plant to plant. Observations were recorded from five randomly selected sample plants in each treatment/replication and observed mean value used for statistical analysis. Characterization of genotypes was also done as per IPGRI (1997) descriptor of Dioscorea spp.

\section{Results and Discussion}

The results of characterization and grouping of genotypes for different morphological characters based on characterization data as per IPGRI descriptor are presented in Table 2 to 4. The collected genotypes show wide range of variability in morphological characteristics. More variability was recorded in genotypes collected from Sukma district of Chhattisgarh followed by Bijapur and Kanker district. 
Table.1 Collection details of indigenous genotypes of Dioscorea bulbifera

\begin{tabular}{|c|c|c|c|c|}
\hline S. No. & Collection No. and Name & Place of Collection & Status of plant (Cultivated/Wild) & Collected Material \\
\hline 1. & IGDb-MTPL-17-1 & Village-Metupalli (Bijapur) & Cultivated (Collected from Badi) & Aerial Tuber \\
\hline 2. & IGDb-BJP-17-2 & Bijapur & Cultivated (Collected from Badi) & Aerial Tuber \\
\hline 3. & IGDb-GDM-17-3 & Geedam (Dantewada) & Cultivated (Collected from Badi) & Aerial Tuber \\
\hline 4. & IGDb-KDNR-17-4 & Kodenar (Bastar) & Cultivated (Collected from Badi) & Aerial Tuber \\
\hline 5. & IGDb-KSKL-17-5 & Keshakl-1(Kondagaon) & Cultivated (Collected from Badi) & Aerial Tuber \\
\hline 6. & IGDb-GRBD-17-6 & Gariyaband & Cultivated (Collected from Badi) & Aerial Tuber \\
\hline 7. & IGDb-UMDH-17-7 & Umradah (Kanker) & Cultivated (Collected from Badi) & Aerial Tuber \\
\hline 9. & IGDb-RJNG-17-9 & Rajnandgaon & Cultivated (Collected from Badi) & Aerial Tuber \\
\hline 10. & IGDb-NGR-17-10 & Nagari (Dhamtari) & Cultivated (Collected from Badi) & Aerial Tuber \\
\hline 11. & IGDb-KNR-17-11 & Kanker & Cultivated (Collected from Badi) & Aerial Tuber \\
\hline 12. & IGDb-MLGD-17-12 & Mulaguga (Sukma) & Cultivated (Collected from Badi) & Aerial Tuber \\
\hline 13. & IGDb-GOLGD-17-13 & Gollaguda (Sukma) & Cultivated (Collected from Badi) & Aerial Tuber \\
\hline 14. & IGDb-KNDR-17-14 & Kondre (Sukma) & Cultivated (Collected from Badi) & Aerial Tuber \\
\hline 15. & IGDb-KDRS-17-15 & Kudukras (Sukma) & Cultivated (Collected from Badi) & Aerial Tuber \\
\hline 17. & IGDb-ARNL-17-17 & Arjunali (Bijapur) & Cultivated (Collected from Badi) & Aerial Tuber \\
\hline 18. & IGDb-MOTT-17-18 & Mottha (Sukma) & Cultivated (Collected from Badi) & Aerial Tuber \\
\hline 19. & IGDb-ATPL-17-19 & Aatpal (Sukma) & Cultivated (Collected from Badi) & Aerial Tuber \\
\hline 20. & IGDb-KNGD-17-20 & Konanguda (Bijapur) & Cultivated (Collected from Badi) & Aerial Tuber \\
\hline 21. & IGDb-NDPL-17-21 & Nedpalli (Sukma) & Cultivated (Collected from Badi) & Aerial Tuber \\
\hline 22. & IGDb-MTR-17-22 & Mater (Sukma) & Cultivated (Collected from Badi) & Aerial Tuber \\
\hline 23. & IGDb-KTGD-17-23 & Kottaguda (Sukma) & Cultivated (Collected from Badi) & Aerial Tuber \\
\hline 24. & IGDb-PSML-17-24 & Puspmalli (Sukma) & Cultivated (Collected from Badi) & Aerial Tuber \\
\hline 25. & IGDb-MHL-17-25 & Mohala (Rajnandgaon) & Cultivated (Collected from Badi) & Aerial Tuber \\
\hline 26. & IGDb-BRMG-17-26 & Bhairamgarh (Bijapur) & Cultivated (Collected from Badi) & Aerial Tuber \\
\hline 27. & IGDb-DMTR-17-27 & Dhamtari & Cultivated (Collected from Badi) & Aerial Tuber \\
\hline 28. & IGDb-BLD-17-28 & Darbari Nawagaon (Balod) & Cultivated (Collected from Badi) & Aerial Tuber \\
\hline 29. & IGDb-MDKMT-17-29 & Mendrakhurd (Ambikapur) & Cultivated (Collected from Badi) & Aerial Tuber \\
\hline 30. & IGDb-MDKDR-17-30 & Mendrakhurd (Ambikapur) & Cultivated (Collected from Badi) & Aerial Tuber \\
\hline
\end{tabular}


Table.2 Morphological characterization of indigenous genotypes of Aerial Yam (Dioscorea bulbifera) as per IPGRI descriptor

\begin{tabular}{|c|c|c|c|c|c|c|c|c|}
\hline \multirow{2}{*}{$\begin{array}{l}\text { S. } \\
\text { N. }\end{array}$} & \multicolumn{8}{|c|}{ A. Leaf characters } \\
\hline & Collection No. and Name & Leaf shape & Leaf position & $\begin{array}{l}\text { Distance between } \\
\text { lobes }\end{array}$ & Petiole colour & $\begin{array}{l}\text { Petiole length in } \\
\text { correlation to leaf } \\
\text { blade }\end{array}$ & $\begin{array}{l}\text { Leaf apex } \\
\text { shape }\end{array}$ & $\begin{array}{c}\text { Leaf } \\
\text { margin }\end{array}$ \\
\hline 1 & IGDb-MTPL-17-1 & Cordate & Opposite & Intermediate & All green with purple at both ends & Long & Acute & Entire \\
\hline 2 & IGDb-BJP-17-2 & Cordate & Opposite & Intermediate & All purplish green with purple at both ends & Medium & Acute & Entire \\
\hline 3 & IGDb-GDM-17-3 & Cordate & Opposite & Intermediate & All green with purple at both ends & Long & Acute & Entire \\
\hline 4 & IGDb-KDNR-17-4 & Cordate & Opposite & Intermediate & All green with purple at both ends & Short & Acute & Entire \\
\hline 5 & IGDb-KSKL-17-5 & Cordate & Opposite & Intermediate & All green with purple at both ends & Short & Acute & Entire \\
\hline 7 & IGDb-UMDH-17-7 & Cordate & Opposite & Intermediate & All purplish green with purple at both ends & Short & Acute & Entire \\
\hline 8 & IGDb-KRB-17-8 & Cordate & Opposite & Intermediate & All purplish green with purple at both ends & Long & Acute & Entire \\
\hline 9 & IGDb-RJNG-17-9 & Cordate & Opposite & Intermediate & All green with purple at both ends & Long & Acute & Entire \\
\hline 10 & IGDb-NGR-17-10 & Cordate & Opposite & Intermediate & All green with purple at both ends & Long & Acute & Entire \\
\hline 11 & IGDb-KNR-17-11 & Cordate & Opposite & Intermediate & All green with purple at both ends & Long & Acute & Entire \\
\hline 12 & IGDb-MLGD-17-12 & Cordate & Opposite & Intermediate & All green with purple at both ends & Long & Acute & Entire \\
\hline 13 & IGDb-GOLGD-17-13 & Cordate & Opposite & Intermediate & All purplish green with purple at both ends & Medium & Acute & Entire \\
\hline 14 & IGDb-KNDR-17-14 & Cordate & Opposite & Intermediate & All purplish green with purple at both ends & Long & Acute & Entire \\
\hline 16 & IGDb-NRNP-17-16 & Cordate & Opposite & Intermediate & All green with purple at both ends & Long & Acute & Entire \\
\hline 17 & IGDb-ARNL-17-17 & Cordate & Opposite & Intermediate & All green with purple at both ends & Long & Acute & Entire \\
\hline 18 & IGDb-MOTT-17-18 & Cordate & Opposite & Intermediate & All green with purple at both ends & Long & Acute & Entire \\
\hline 19 & IGDb-ATPL-17-19 & Cordate & Opposite & Intermediate & All green with purple at both ends & Long & Acute & Entire \\
\hline 2 & IGDb-KNGD-17-20 & Cordate & Opposite & Intermediate & All green with purple at both ends & Long & Acute & Entire \\
\hline 21 & IGDb-NDPL-17-21 & Cordate & Opposite & Intermediate & All green with purple at both ends & Long & Acute & Entire \\
\hline 22 & IGDb-MTR-17-22 & Cordate & Opposite & Intermediate & All purplish green with purple at both ends & Long & Acute & Entire \\
\hline 23 & IGDb-KTGD-17-23 & Cordate & Opposite & Intermediate & All purplish green with purple at both ends & Long & Acute & Entire \\
\hline 24 & IGDb-PSML-17-24 & Cordate & Opposite & Intermediate & All purplish green with purple at both ends & Long & Acute & Entire \\
\hline 25 & IGDb-MHL-17-25 & Cordate & Opposite & Intermediate & All green with purple at both ends & Long & Acute & Entire \\
\hline 26 & IGDb-BRMG-17-26 & Cordate & Opposite & Intermediate & All green with purple at both ends & Long & Acute & Entire \\
\hline 27 & IGDb-DMTR-17-27 & Cordate & Opposite & Intermediate & All green with purple at both ends & Medium & Acute & Entire \\
\hline 28 & IGDb-BLD-17-28 & Cordate & Opposite & Intermediate & All green with purple at both ends & Long & Acute & Entire \\
\hline 29 & IGDb-MDKMT-17-29 & Cordate & Opposite & Intermediate & All green with purple at both ends & Medium & Acute & Entire \\
\hline 30 & IGDb-MDKDR-17-30 & Cordate & Opposite & Intermediate & All green with purple at both ends & Long & Acute & Entire \\
\hline
\end{tabular}


Table.3 Morphological characterization of indigenous genotypes of Aerial Yam (Dioscorea bulbifera) as per IPGRI descriptor

\begin{tabular}{|c|c|c|c|c|c|c|c|}
\hline \multirow[t]{2}{*}{ S. No. } & \multicolumn{7}{|c|}{ B. Vine, plant and aerial Tuber characters } \\
\hline & Collection No. and Name & $\begin{array}{c}\text { Aerial tuber } \\
\text { Texture }\end{array}$ & $\begin{array}{l}\text { Aerial tuber skin } \\
\text { colour }\end{array}$ & $\begin{array}{l}\text { Aerial tuber } \\
\text { Shape }\end{array}$ & $\begin{array}{c}\text { No. of underground } \\
\text { tuber }\end{array}$ & $\begin{array}{l}\text { Twinning } \\
\text { direction }\end{array}$ & $\begin{array}{c}\text { Present/ absent of } \\
\text { spine }\end{array}$ \\
\hline 1. & IGDb-MTPL-17-1 & Wrinkled & Dark Brown & Round & 1 & Clockwise & Absent \\
\hline 2. & IGDb-BJP-17-2 & Rough & Dark Brown & Round & 1 & Clockwise & Absent \\
\hline 3. & IGDb-GDM-17-3 & Wrinkled & Dark Brown & Round & 1 & Clockwise & Absent \\
\hline 4. & IGDb-KDNR-17-4 & Smooth & Light Brown & Round & 1 & Clockwise & Absent \\
\hline 5. & IGDb-KSKL-17-5 & Smooth & Dark Brown & Round & 1 & Clockwise & Absent \\
\hline 6. & IGDb-GRBD-17-6 & Smooth & Light Brown & Round & 1 & Clockwise & Absent \\
\hline 7. & IGDb-UMDH-17-7 & Wrinkled & Light Brown & Round & 1 & Clockwise & Absent \\
\hline 8. & IGDb-KRB-17-8 & Rough & Dark Brown & Round & 1 & Clockwise & Absent \\
\hline 9. & IGDb-RJNG-17-9 & Rough & Dark Brown & Round & 1 & Clockwise & Absent \\
\hline 10. & IGDb-NGR-17-10 & Wrinkled & Light Brown & Round & 1 & Clockwise & Absent \\
\hline 11. & IGDb-KNR-17-11 & Rough & Dark Brown & Round & 1 & Clockwise & Absent \\
\hline 12. & IGDb-MLGD-17-12 & Rough & Dark Brown & Round & 1 & Clockwise & Absent \\
\hline 13. & IGDb-GOLGD-17-13 & Wrinkled & Dark Brown & Round & 1 & Clockwise & Absent \\
\hline 14. & IGDb-KNDR-17-14 & Rough & Dark Brown & Round & 1 & Clockwise & Absent \\
\hline 15. & IGDb-KDRS-17-15 & Rough & Dark Brown & Round & 1 & Clockwise & Absent \\
\hline 16. & IGDb-NRNP-17-16 & Wrinkled & Light Brown & Round & 1 & Clockwise & Absent \\
\hline 17. & IGDb-ARNL-17-17 & Rough & Dark Brown & Round & 1 & Clockwise & Absent \\
\hline 18. & IGDb-MOTT-17-18 & Wrinkled & Light Brown & Round & 1 & Clockwise & Absent \\
\hline 19. & IGDb-ATPL-17-19 & Rough & Dark Brown & Round & 1 & Clockwise & Absent \\
\hline 20. & IGDb-KNGD-17-20 & Rough & Dark Brown & Round & 1 & Clockwise & Absent \\
\hline 21. & IGDb-NDPL-17-21 & Rough & Dark Brown & Round & 1 & Clockwise & Absent \\
\hline 22. & IGDb-MTR-17-22 & Wrinkled & Light Brown & Round & 1 & Clockwise & Absent \\
\hline 23. & IGDb-KTGD-17-23 & Wrinkled & Light Brown & Round & 1 & Clockwise & Absent \\
\hline 24. & IGDb-PSML-17-24 & Wrinkled & Light Brown & Round & 1 & Clockwise & Absent \\
\hline 25. & IGDb-MHL-17-25 & Rough & Dark Brown & Round & 1 & Clockwise & Absent \\
\hline 26. & IGDb-BRMG-17-26 & Rough & Dark Brown & Round & 1 & Clockwise & Absent \\
\hline 27. & IGDb-DMTR-17-27 & Wrinkled & Light Brown & Round & 1 & Clockwise & Absent \\
\hline 28. & IGDb-BLD-17-28 & Rough & Dark Brown & Round & 1 & Clockwise & Absent \\
\hline 29. & IGDb-MDKMT-17-29 & Wrinkled & Light Brown & Round & 1 & Clockwise & Absent \\
\hline 30. & IGDb-MDKDR-17-30 & Rough & Dark Brown & Round & 1 & Clockwise & Absent \\
\hline
\end{tabular}


Table.4 Frequency distribution of quantitative traits of Dioscorea bulbifera

\begin{tabular}{|c|c|c|c|}
\hline S.N. & Quantitative characters & Index and description adopted & Frequency $(\%)$ \\
\hline 1. & Leaf shape & Cordate & 100 \\
\hline 2. & Leaf position & Opposite & 100 \\
\hline 3. & Distance between lobes & Intermediate & 100 \\
\hline \multirow[t]{2}{*}{4.} & \multirow[t]{2}{*}{ Petiole colour } & All purplish green with purple at both ends & 73.33 \\
\hline & & All green with purple at both ends & 26.67 \\
\hline \multirow[t]{3}{*}{5.} & \multirow{3}{*}{$\begin{array}{l}\text { Petiole length in } \\
\text { correlation to leaf blade }\end{array}$} & Short $(<2 \mathrm{~cm})$ & 13.33 \\
\hline & & Medium $(=2 \mathrm{~cm})$ & 13.33 \\
\hline & & Long $(>2 \mathrm{~cm})$ & 73.33 \\
\hline 6. & Leaf apex shape & Acute & 100 \\
\hline 7. & Leaf margin & Entire & 100 \\
\hline \multirow[t]{3}{*}{8.} & \multirow[t]{3}{*}{ Aerial tuber Texture } & Smooth & 10 \\
\hline & & Wrinkled & 40 \\
\hline & & Rough & 50 \\
\hline \multirow[t]{2}{*}{9.} & \multirow[t]{2}{*}{ Aerial tuber skin colour } & Light brown & 36.67 \\
\hline & & Dark brown & 63.33 \\
\hline 10. & Aerial tuber Shape & Round & 100 \\
\hline 11. & Present/ absent of spine & Absent & 100 \\
\hline 12. & Twinning direction & Present & 100 \\
\hline
\end{tabular}

\section{Leaf and petiole character}

All the collected genotypes of Dioscorea bulbifera exhibits cordate leaf shape, opposite leaf position, entire leaf margin, acute apex shape and distance between lobes is intermediate. No. of leaves/plant ranged from 12.43 to 23.94. Highest number of leaves was recorded in genotype IGDb-MHL-17-25 and lowest in IGDb-BJP-17-2. Highest leaf length $(14.09 \mathrm{~cm})$ was observed in genotypes IGDbNGR-17-10 and lowest leaf length $(8.51 \mathrm{~cm})$ was recorded in IGDb-MHL-17-25.

Genotypes were categorized into two groups on the basis of petiole colour. Petiole colour varied from all green with purple at both ends and all purplish green with purple at both ends with the frequency of $26.67 \%$ and $73.33 \%$ respectively. Genotypes were categorized in to three groups i.e. short, medium and large petiole length in correlation to leaf blade. The highest frequency was observed in long petiole length in correlation to leaf blade $(73.3 \%)$ followed by short (13.3\%) and medium (13.33\%).

\section{Stem and plant character}

All the collected genotypes exhibit clockwise twinning habit and spineless stem. No spine was recorded in collected genotypes; however stem length, internode number to $1^{\text {st }}$ branching differs significantly.

\section{Tuber character}

Genotypes are categorized into three groups on the basis of aerial tuber texture i.e. smooth, wrinkled and rough. Highest frequency $(50 \%)$ was recorded as rough aerial tuber texture followed by wrinkled (40\%) and lowest frequency $(10 \%)$ was recorded as smooth aerial tuber texture. All the collected Dioscorea bulbifera genotypes exhibit round shape aerial tuber having single underground tuber. The collected genotypes shows dark brown and light brown aerial tuber skin colour with the frequency of $63.33 \%$ and $36.67 \%$ respectively.

Dioscorea bulbifera globally known as aerial yam or air potato is distinguished from the other Dioscorea species by having special aerial bulbils which appear at the base of the leaf petioles (Croxton et al., 2011; Silva et al., 2016). The range and mean performance showed the presence of considerable amount of variability among the accessions. For instance, 
bulbils fresh yield varied from 4.39 to 14.57 tones/ha, tuber yield varied from 2.0 to 8.22 tones/ha, number of bulbils varied from 43.66 to $98.67 /$ plot, bulbils length varied from 5.33 to $9.0 \mathrm{~cm}$ and tuber diameter varied from 5.64 to $9.15 \mathrm{~cm}$ (Mulualem and Mohammed, 2012).

Mulualem and WeldeMichae, G. (2013) characterized Dioscorea Species and observed that, the bulbils skin color of the accessions ranged from light brown $(23.40 \%)$ to dark brown $(40.43 \%)$, whereas bulbils were round $(38.30 \%)$, oval $(34.04 \%)$, elongate $(21.28 \%)$ and irregular $(6.38 \%)$ in shape. In this study, accessions showed three different bulbils surface textures: smooth $(61.70 \%)$, wrinkled $(14.90 \%)$ and rough $(23.40 \%)$. Similarly, it is in agreement with the works of Muluneh et al., (2006) who reported that there is a wide range of variability of tubers among in Ethiopia. Furthermore, similar result Dioscorea species was reported by Asfaw (2006) in Taro and Woyessa (2006) in Plectranthus edulis.

\section{References}

Annonymous. 2005. Annual Report of the National Bureau of Plant Genetic Resources 2005 - 2006, NBPGR, Pusa Campus, New Delhi, India, 148 pp.

Asfaw K. 2006. Characterization and divergence analysis of some Ethiopian taro (Colocasia esculenta (L.) accessions. M.Sc thesis, Alemaya University, Alemaya.

Croxton M.D., Andreu M.A., Williams D.A., Overholt W.A., Smith J.A. 2011. Geographic Origins and Genetic Diversity of Air-Potato (Dioscorea bulbifera) in Florida. Invasive Plant Science and Management, 4: 22-30.
Muluneh, T. 2006. Assessing diversity in yam (from Ethiopia based on morphology, AFLP Dioscorea spp.) marker and tuber quality and farmers' management of landraces. Ph.D. thesis, George -August University. Germany

Mulualem, T. and Mohammed, H. 2012. Genetic variability and association among yield and yield related traits in Aerial Yam /Dioscorea bulbifera (L.)/ Accessions at South western Ethiopia. Journal of Natural Sciences Research, 2:.9.

Mulualem T. and WeldeMichae G. 2013. Agronomical Evaluation of Aerial Yam/ Dioscorea bulbiferal Accessions collected from South and Southwest Ethiopia. Greener Journal of Agricultural Sciences 3 (9), pp. 693-704.

Silva D.M., Siqueira M.V.B.M., Carrasco N.F., Mantello C.C., Nascimento W.F., Veasey E.A. 2016. Genetic diversity among air yam (Dioscorea bulbifera) varieties based on single sequence repeat markers. Genetics and molecular research, 2016, 15, gmr.15027929.

Thomas, T. A and P. N. Mathur. 1991. Germplasm Evaluation and Utilization. In: Plant Genetic Resources Conservation and Management. R. S. Paroda and R. K. Arora (eds.).Published by the International Board for Plant Genetic Resources, Regional Office for South and Southeast Asia, NBPGR, Pusa Campus, New Delhi 110012, India. pp. 149-18 1.

Woyessa, G. 2006. Morphological characterization and divergence analysis of (Vatke) Plectranthus edulis. Agnew collection in Ethiopia. M.Sc. thesis, Presented to School of Graduate Studies, Hawassa University, Awassa.

\section{How to cite this article:}

Kamal Narayan, J. Singh, Deo Shankar, R. Gayen, N. Mehta and Saxena, R. R. 2020. Collection and Characterization of Indigenous Dioscorea bulbifera Genotypes of Chhattisgarh. Int.J.Curr.Microbiol.App.Sci. 9(09): 3463-3469. doi: https://doi.org/10.20546/ijcmas.2020.909.430 